\title{
Silicon-processed plastic micropyramids for brightness enhancement applications
}

\author{
Liwei Lin †\&, T K Shia† and C-J Chiu† \\ $\dagger$ Department of Mechanical Engineering and Applied Mechanics, University of Michigan, \\ USA \\ $\$$ Institute of Applied Mechanics, National Taiwan University, Taipei, Taiwan \\ E-mail: lwlin@me.berkeley.edu
}

Received 15 February 2000

Abstract. A combination of silicon anisotropic etch and mechanical hot embossing techniques is used to demonstrate the feasibility of manufacturing plastic micropyramids. These micropyramids have the desirable optical characteristics to enhance the brightness of LCD (liquid crystal displays) in battery-powered laptop computers, personal TVs and camcorders. Silicon mold inserts, 4-inch in diameter, are first micromachined and used directly in the micro hot embossing process by applying plastic sheets made of polymethyl methacrylate (PMMA) or polyvinyl chloride (PVC). Micropyramids which have base widths of $30 \mu \mathrm{m}$, heights of $21 \mu \mathrm{m}$ and apex angles of $70^{\circ}$ are hot embossed on the surface of plastic sheets. The fabricated plastic films are tested optically and the results show that up to $20 \%$ brightness enhancement within the front viewing angles of $\pm 35^{\circ}$ have been achieved. An optical model has been established to simulate the brightness enhancement effects and it is concluded that the optimal pyramid angle for the brightness enhancement application is $90^{\circ}$.

\author{
Nomenclature \\ $d$ distance \\ $h$ the height of the micropyramid \\ $\vec{m} \quad$ unit vector normal to the interface plane \\ $n$ refraction index \\ $\vec{t} \quad$ unit vector representing the direction of light \\ $\theta$ angle
}

\section{Subscript and superscipt}

$\begin{array}{ll}b & \text { perpendicular to } \vec{t}_{m} \text { and } \vec{m} \\ i & \text { incident light } \\ j & \text { number of inclined plane of a pyramid } \\ m & \text { perpendicular to } \vec{t} \text { and } \vec{m} \\ x, y, z & \text { coordinates in the Cartesian coordinate system } \\ c r i & \text { critical } \\ \text { fle } & \text { reflection } \\ \text { fra } & \text { refraction }\end{array}$

\section{Introduction}

Brightness enhancement films can be expected to have broad applications for battery-powered displays in consumer electronics devices such as laptop computers, personal TVs and camcorders. These passive films, as illustrated in

$\S$ Current address: 5126 Etcheverry Hall, Department of Mechanical Engineering, University of California, Berkeley, CA 94720-1740, USA.

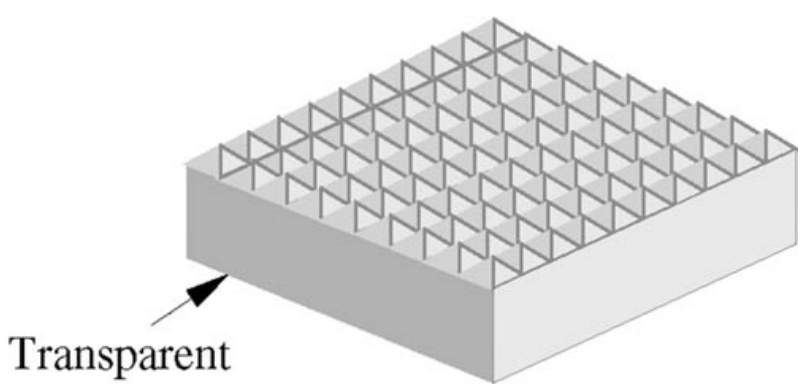

Figure 1. The schematic diagram of brightness enhancement film.

figure 1, are made of plastic materials and are optically transparent. They can save unnecessary power consumption by redirecting and redistributing the incident light coming from the back side of the films to the front side and within confined and concentrated viewing angles.

The combination of precision mechanical machining and hot embossing can provide many fine manufacturing processes. For example, fine microstructures can be first defined on metal sheets by precision diamond cutting and plastic replicas are then duplicated by using the molding process. In order to improve the optical efficiency of these films, it is important to fabricate mold inserts that have extremely smooth surfaces. The batch processing capability in IC (integrated circuit) technologies provides distinct manufacturing advantages over conventional machining methods. For example, many advanced manufacturing 
possibilities have been demonstrated in the field of microelectromechanical systems (MEMS), including the construction of three-dimensional microstructures by means of silicon micromachining [1].

Previously, diamond and other mechanical machining technologies have been used to make microcars [2], microchannels or micropyramids $[3,4]$. It is difficult to maintain the surface roughness of microstructures that are made by these conventional, mechanical systems. Furthermore, mass production is a big problem. Processes that combine micromachining and plastic molding technologies have been introduced recently to address these shortcomings. These are the LIGA [5], the DEEMO (Dry Etching, Electroplating and MOlding) process [6], the microlens process $[7,8]$, the conventional CD-injection molding technique $[9,10]$, and the thermoplastic replication [11] processes. These processes use the advantage of micromachining to build fine mold inserts and use the molding process as the massive, but inexpensive manufacturing tool.

This paper presents a combined process of silicon anisotropic etching and micro hot embossing to manufacture micropyramids for brightness enhancement applications in consumer electronics. A 4-inch wafer is directly used as the mold insert to achieve large-area manufacturing. Microstructures with the shape of pyramids are built on top of plastic films to redirect the light and enhance the brightness. A theoretical model is developed to simulate the optical effects and the fabricated films are tested under a backlight source to characterize the brightness enhancement effects.

\section{Operation principle and manufacturing methods}

The optical principle of a pyramid-shaped structure is illustrated in figure 2 . The emitting light from the bottom of the pyramids can be divided into three major parts: (a) direct recycle, (b) effective refraction, and (c) indirect recycle. The path of the direct recycling part is illustrated in figure 2(a) where the incoming light is reflected back and recycled. Figure 2(b) shows the effective refraction when the light is refracted out of the film and emitted at the front of the film. The third part is shown in figure 2(c) where the light is reflected first and then refracted by the film. The exit angles are such that the light will hit another pyramid and go back to the film as the indirect recycling part. In addition to the three major parts as described, light may also travel through several pyramids via repeated reflection and refraction. The overall effect is the redirection and redistribution of light after the light passes through these pyramids, such that the intensity of light within the viewing angles of $\pm 35^{\circ}$ increases. Because this mechanism is passive, enhanced brightness is accomplished while no extra input energy is required.

A process which combines micromachining technologies and plastic molding has been developed to fabricate films with micropyramids on top, as shown in figure 3 . The silicon micromachining process uses only one mask. First, $\mathrm{SiO}_{2}$ is thermally grown on top of the (100)-oriented silicon wafer. It is then passed through the lithography process by using the standard UV light exposure as seen in figure 3(a). The patterns on the mask are then transferred onto the photoresist on top of the (100)-oriented silicon wafer. The minimum

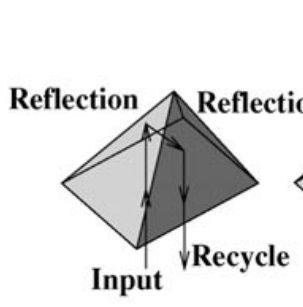

(a)

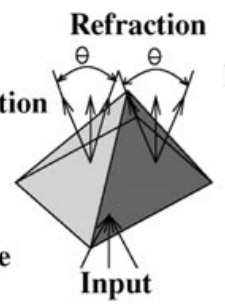

(b)

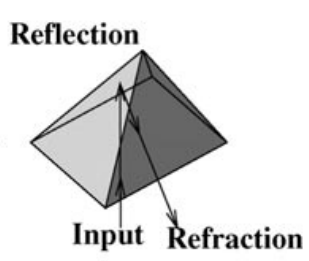

(c)
Figure 2. The optical principle showing three possible cases of the emitting light: (a) direct recycle; (b) effective refraction; (c) indirect recycle.

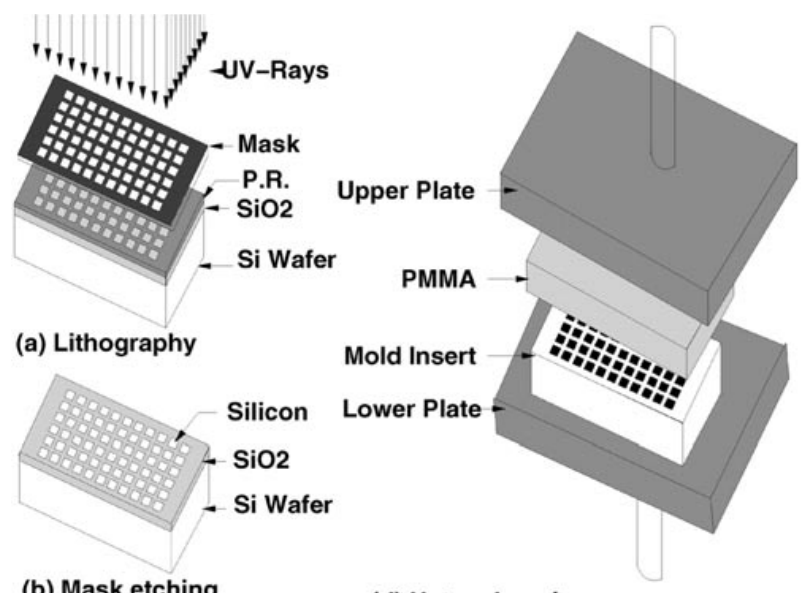

(b) Mask etching

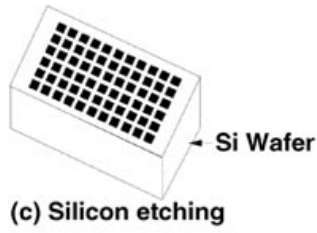

(c) Silicon etching (d) Hot embossing

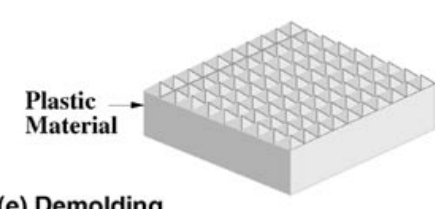

(e) Demolding
Figure 3. The fabrication sequences.

width used in the mask is $2 \mu \mathrm{m}$ in this trial run and the whole 4-inch wafer is divided into several areas. Each area has a size of $1 \mathrm{~cm}^{2}$ and has the same pyramid arrays with base width of either $100 \mu \mathrm{m}$ or $30 \mu \mathrm{m}$. The unwanted silicon dioxide layer is then removed by using HF solution. The silicon anisotropic etching process then follows so as to define the shape of the micropyramids into the silicon wafer [12]. Figure 3(b) applies after these steps. The masking material is then removed such that the patterned microstructures are on the top of the silicon wafer as shown in figure 3(c). The whole silicon wafer is then directly used as the master mold insert in a hot embossing machine as illustrated in figure 3(d). Plastic material that generally has the glass transition point at a medium temperature is used in the hot embossing process. Fine micropatterns are transferred onto the plastic material as shown in figure 3(e). This process can be repeated to duplicate plastic microstructures on top of plastic materials inexpensively by batch processing.

The molding process can be conducted either in the laboratory or in a commercial environment [10]. For the laboratory process [13], hot embossing takes place under vacuum (about $0.1 \mathrm{mbar}$ ) to realize complete filling of the mold with PMMA of $300 \mu \mathrm{m}$ in thickness. After evacuation, 
the mold is heated up to the molding temperature of $200^{\circ} \mathrm{C}$, which lies above the glass transition temperature of the polymer. Higher temperatures are favorable because the lower viscosity of the plastic facilitates the molding process. During the molding process, a force of $10 \mathrm{kN}$ is applied. After molding, the polymer is cooled down to below the glass transition temperature. A molding force is maintained during cooling in order to preserve the plastic microstructures from shrinking. As soon as the predetermined temperature has been reached, the microstructures can be separated from the mold insert. The high molding forces introduce a new problem in the de-molding process: for small forces demolding takes place automatically upon cooling to room temperature. In the case of a high molding force, this normal procedure is no longer sufficient. The combination of molded films and the mold insert have to be put into a refrigerator at a temperature of around $0^{\circ} \mathrm{C}$. A water film between the mold and the polymer has to be introduced to make use of the additional capillary forces for separation.

For the commercial hot embossing process [10], a custom-made hot embossing machine is used. The silicon mold insert masters are first deposited with a thin layer of silver as the seed layer for electroplating. Nickel is then electroplated on top of the silicon master until its thickness reaches about $300 \mu \mathrm{m}$. The electroplating process is conducted twice to duplicate the same downward micropyramids as in the original silicon mold inserts. The electroplated nickel plate is then used as the mold insert for hot embossing. In this case, the overall process takes only about $1 \mathrm{~min}$ and PVC is used as the plastic material. For every mold insert with different microstructures and different molding machines, adequate hot embossing parameters such as force, temperature and time have to be determined as described previously [10,13].

Figure 4 shows a scanning electron microscope (SEM) micrograph of fabricated arrays of plastic micropyramids made of PMMA. The quality and repeatability of the process can be demonstrated from this figure. These micropyramids have a base width of $30 \mu \mathrm{m}$ and a height of about $21 \mu \mathrm{m}$. The enlarged SEM microphoto shown in figure 5 demonstrates that the surfaces of these micropyramids are very smooth and no defects can be identified in this microphoto. Surface roughness measurements by atomic force microscope (AFM) reveal that (111) surfaces of this fabricated micropyramid have a mean roughness of less than $10 \mathrm{~nm}$ [10]. Figure 6 shows a whole silicon mold insert (right) and a hot embossed plastic duplicate (left), respectively. The silicon wafer is 4 inch in diameter and the plastic duplicate is a little smaller due to the limitation of our lithography machine. This photo demonstrates the feasibility of making largearea hot embossing films, which are desirable for brightness enhancement in practical applications.

\section{Theoretical model}

Micropyramids fabricated by anisotropic silicon etching have four exposed 111 surfaces [12] which form the four inclined planes of a pyramid. The base plane of the pyramid can be defined by four base points. If $h$ is the height of the pyramid in a Cartesian coordinate system, the four base points can be

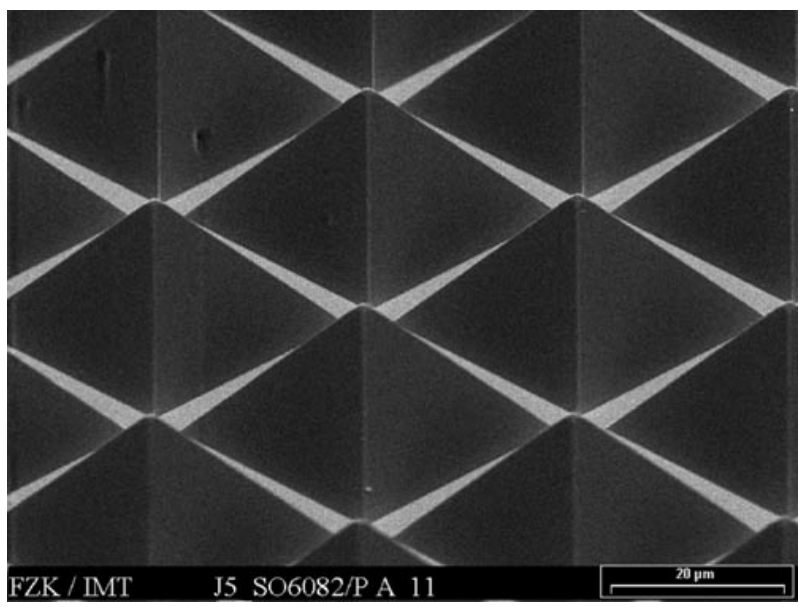

Figure 4. A SEM microphoto showing array of micropyramids.

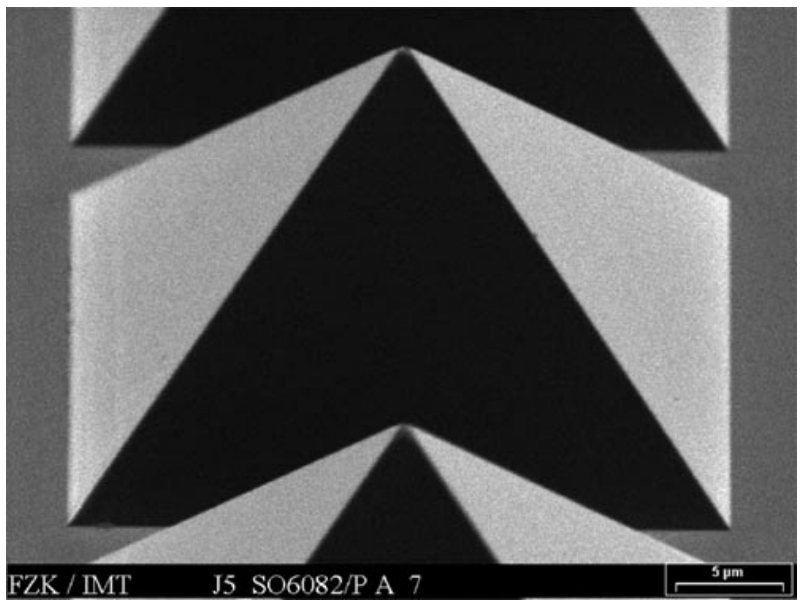

Figure 5. A close view of a plastic micropyramid.

represented by $(h, 0,0),(0, h, 0),(-h, 0,0),(0,-h, 0)$ and the apex point is represented by $(0,0, h)$. The apex angle of the pyramid will be about $70^{\circ}$ in this case. In order to build an optical model to simulate light propagation in the pyramids, equations representing the four inclined planes are first derived as

$$
m_{x}^{j} x+m_{y}^{j} y+m_{z}^{j} z-m_{z}^{j} h=0 \quad(j=1,2,3,4)
$$

where $\left(m_{x}^{j}, m_{y}^{j}, m_{z}^{j}\right)$ is the unit vector normal to the $j$ th inclined plane.

Point light sources from the base plane are uniformly distributed when the pyramid is placed on top of a backlight source. Theoretically, the emitting light will intersect all four inclined planes if the angle of the emitting light is not parallel to any of these planes. The distance from the light source to the intersection point on the $j$ th inclined plane can be calculated as

$$
d^{j}=-\frac{m_{x}^{j} x+m_{y}^{j} y+m_{z}^{j} z-m_{z}^{j} h}{m_{x}^{j} t_{x}+m_{y}^{j} t_{y}+m_{z}^{j} t_{z}}
$$

where $\left(t_{x}, t_{y}, t_{z}\right)$ is the unit vector representing the emitting direction of the light. The actual inclined plane that the light hits is the one that has a minimal positive distance. 


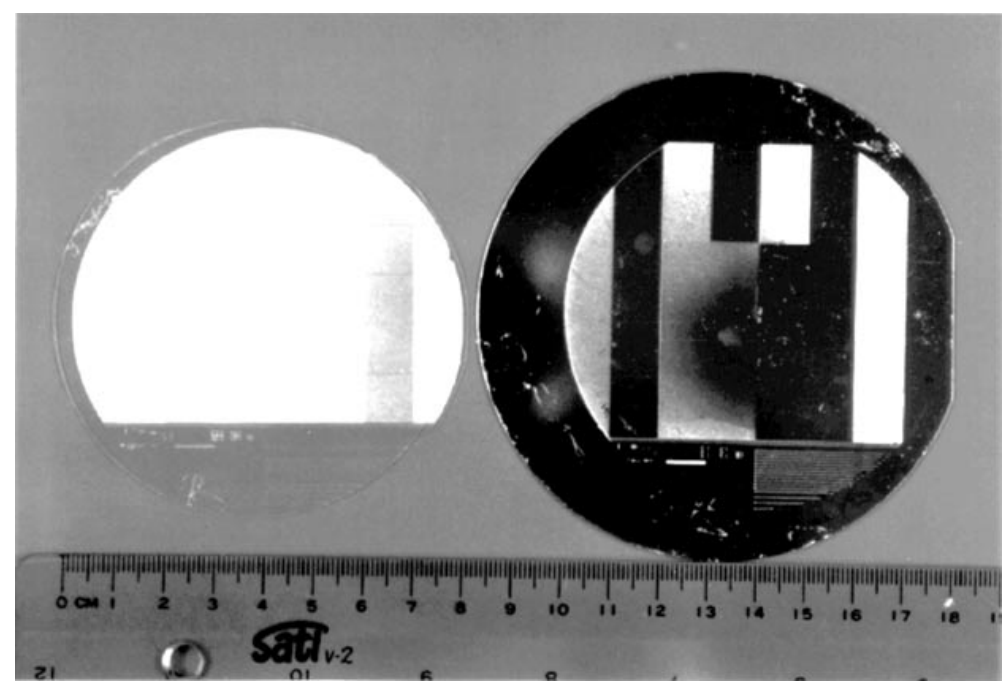

Figure 6. A whole 4-inch silicon mold insert (right) and fabricated plastic film (left).

Once the inclined plane is determined, Snell's law is applied to determine the new propagation direction [14]. If the light is traveling to a less dense medium such as from plastic material to air, either refraction or total reflection may occur. The critical angle for total reflection is represented as

$$
\theta_{c r i}=\sin ^{-1}\left(\sin \left(\frac{\pi}{2}\right)\left(\frac{n_{f r a}}{n_{i}}\right)\right)
$$

where $n_{i}$ and $n_{f r a}$ are the refraction indexes of the medium at the incident side $(i)$ and at the refraction side ( $f r a)$, respectively. When the incident angle is smaller than the critical angle, refraction occurs; otherwise, reflection occurs.

The new unit vector of the reflected light $\left(\vec{t}_{f l e}\right)$ or refracted light $\left(\vec{t}_{\text {fra }}\right)$ is determined by introducing two new vectors as shown in figure 7 . The normal unit vector, $\vec{t}_{m}$, is defined as the unit vector that is perpendicular to both the incident light, $\vec{t}$, and the normal vector of the interface plane, $\vec{m}$. The bi-normal unit vector, $\vec{t}_{b}$, is defined as the unit vector that is perpendicular to the new normal unit vector $\left(\vec{t}_{m}\right)$ and the normal vector of the interface plane $\vec{m}$. They can be derived mathematically as

$$
\begin{gathered}
\vec{t}_{m}=\frac{\vec{t} \times \vec{m}}{|\vec{t} \times \vec{m}|} \\
\vec{t}_{b}=\frac{\vec{t}_{m} \times \vec{m}}{\left|\vec{t}_{m} \times \vec{m}\right|} .
\end{gathered}
$$

The incident angle can be calculated as

$$
\theta_{i}=\cos ^{-1}(-\vec{t} \cdot \vec{m})
$$

When total reflection occurs, the new propagation direction, $\vec{t}_{f l e}$, is calculated by solving the following equation set:

$$
\begin{gathered}
\vec{t}_{f l e} \cdot \vec{t}_{b}=\vec{t} \cdot \vec{t}_{b} \\
\vec{t}_{f l e} \cdot \vec{m}=-\vec{t} \cdot \vec{m} \\
\vec{t}_{f l e} \cdot \vec{t}_{m}=0 .
\end{gathered}
$$

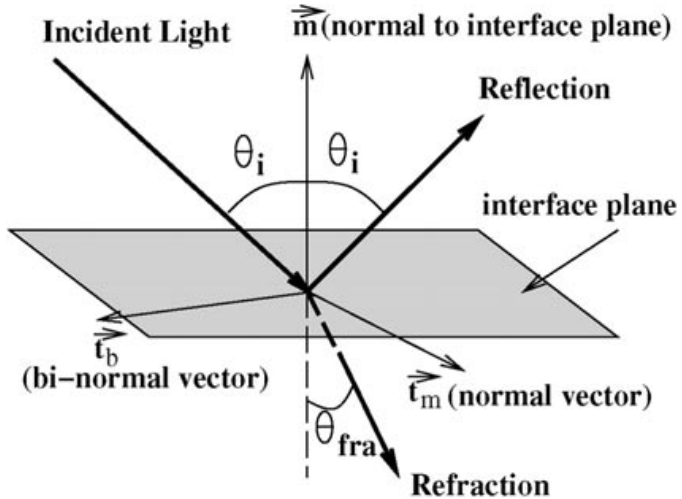

Figure 7. Illustration of optical terms defined in the paper.

When refraction occurs, the new propagation direction, $\vec{t}_{\text {fra }}$, is calculated by solving the following equation set:

$$
\begin{gathered}
\vec{t}_{f r a} \cdot \vec{m}=-\cos \left(\theta_{f r a}\right) \\
\vec{t}_{f r a} \cdot \vec{t}_{m}=0 \\
\vec{t}_{f r a} \cdot \vec{t}_{b}=\cos \left(\frac{\pi}{2}+\theta_{f r a}\right)
\end{gathered}
$$

where

$$
\theta_{f r a}=\sin ^{-1}\left(\sin \left(\theta_{i}\right) \frac{n_{i}}{n_{f r a}}\right)
$$

The above vectors are determined to simulate light propagation in the micropyramids. Light rays that are refracted out as the emitting light to the front of the film are collected and the energy flux at different angles is calculated. It is noted that a small portion of the light rays may actually go through several reflection and refraction processes through several micropyramids before eventually being refracted out. The same principle has been used to analyze pyramids that have apex angles other than $70^{\circ}$ in order to determine the best shape of pyramids for brightness enhancement applications. 


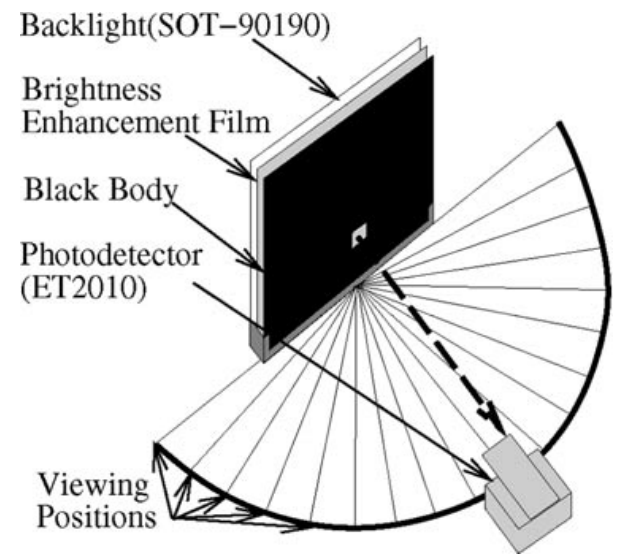

Figure 8. Experimental set-up for the optical test.

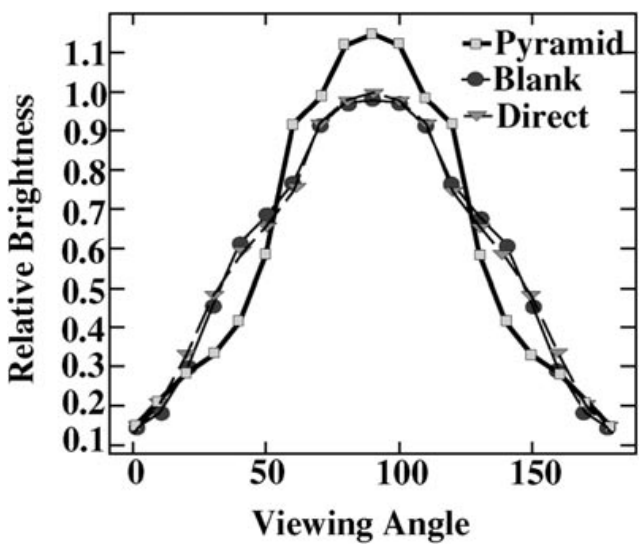

Figure 9. Optical measurements for PMMA films made by the laboratory hot embossing process.

\section{Results and discussions}

Fabricated plastic films that have micropyramids on their surfaces are placed on top of a backlight source [15] for optical measurements. The experimental set-up is shown in figure 8 , where a black body is put in front of the plastic film with a small opening of $1 \mathrm{~cm} \times 1 \mathrm{~cm}$. The power intensity of outgoing light rays is measured at different viewing angles $100 \mathrm{~cm}$ away from the opening. Direct measurements from the backlight (without any films) is used as the base line and the light intensity at $90^{\circ}$ is the base point for calculating relative brightness. Blank plastic sheets (without micropyramids) and plastic films with micropyramids are placed on top of the backlight and optical data are collected for each case.

Figure 9 shows the experimental results for PMMA films made by the laboratory process. It is observed that when the blank PMMA films are in place, the distributions of relative brightness are very close to those from the direct measurement of backlight. The relative brightness is actually a little lower when PMMA films are in place, since the transmission rate of light for PMMA is about $98 \%$. When the micropyramid arrays are tested, the brightness within the viewing angles of $90^{\circ} \pm 35^{\circ}$ is enhanced as shown. The maximum enhancement is about $15 \%$ at the center. On the other hand, the brightness that is outside the viewing angle decreases as the result of light redistribution. Similar results for PVC films

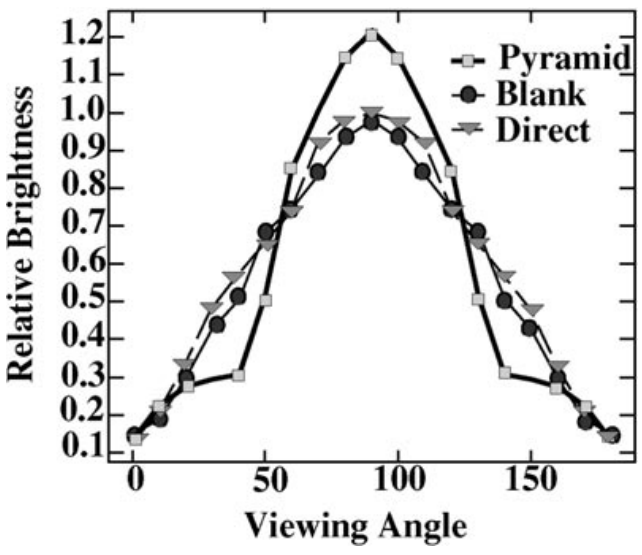

Figure 10. Optical measurements for PVC films made by the commercial hot embossing process.

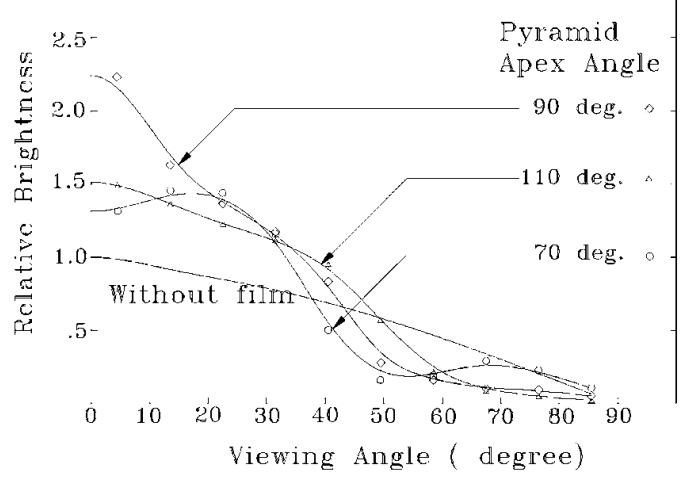

Figure 11. Optical simulation results of relative brightness at different viewing angles.

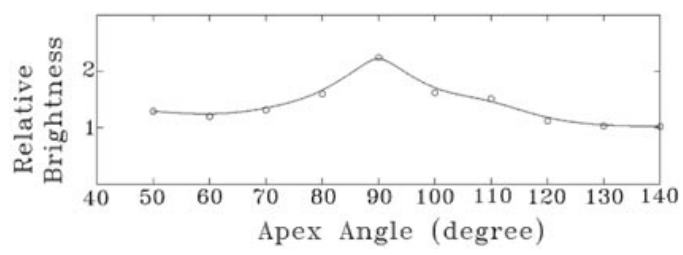

Figure 12. Simulation results of relative brightness at the center of the viewing direction with respect to different apex angles of the pyramids.

made by the commercial hot embossing process are recorded as shown in figure 10. These plastic films are made of PVC and the transmission rate of light for PVC is about $96 \%$. The maximum brightness enhancement occurs at the center and about $20 \%$ brightness enhancement has been achieved.

A computer simulation program has been developed based on the optical theory. Light rays coming out of the backlight source are assumed to follow Lambert's law [14] and are monochromatic and incoherent. The simulation program uses uniformly distributed point light sources on the base of a pyramid to simulate backlight. A total of 181 points are used on one-eighth of a pyramid due to symmetry. It is also assumed that energy absorbed by the film is neglected. Figure 11 shows the simulation results for pyramids with apex angles of $70^{\circ}, 90^{\circ}$ and $110^{\circ}$. Pyramids with $90^{\circ}$ apex angle provide more than $100 \%$ brighter effects near the viewing 
angle at $0^{\circ}$. Pyramids with $70^{\circ}$ apex angle, on the other hand, seem to increase $30-50 \%$ within the viewing angle of $\pm 35^{\circ}$. In order to characterize the effects of apex angles, a series of simulations have been conducted and the relative brightnesses around the zero viewing angle are plotted in figure 12. It is found that pyramids with apex angle of $90^{\circ}$ have the best brightness enhancement effect.

Anisotropic silicon etching reported in this paper is capable of making pyramids with apex angles of $70^{\circ}$. The simulation results seem to predict a better enhancement effect than the experiments as shown in figures 10 and 11 . The discrepancy comes from the fact that several effects are neglected in the simulation program. First, energy absorbed by the pyramid films has not been considered in the simulation. Second, partial reflection or refraction has been neglected in the theoretical calculation. Third, surfaces of the pyramids have been assumed to be perfect in theory but this is not the case for real pyramids. Finally, and most importantly in this case, the lines separating the pyramids have been neglected in the simulation. These lines have a width of $2 \mu \mathrm{m}$ and will have no brightness enhancement effect. All of the above factors contribute to the overestimation of brightness enhancement effects in the simulation program. They can be included in the optical model if more detailed simulations are desired. On the issue of apex angle, the simulation results reveal that pyramids with $90^{\circ}$ apex angle would give the best performance for brightness enhancement. However, $90^{\circ}$ micropyramids made by silicon processing have not been demonstrated. Previously, $45^{\circ}$ mirrors have been fabricated by silicon micromachining technology [16] that may form one inclined plane for a $90^{\circ}$ micropyramid.

\section{Conclusions}

Plastic micropyramids based on the manufacturing technologies of silicon anisotropic etching and micro hot embossing processes have been demonstrated. These micropyramids are defined by silicon (111) planes with base widths of $30 \mu \mathrm{m}$ and heights of $21 \mu \mathrm{m}$. Both PMMA films fabricated by a laboratory process and PVC films made by a commercial embossing process have been successfully manufactured and optically tested. These transparent films have been shown to enhance the brightness of a backlight source by up to $20 \%$ within the viewing angle of $\pm 35^{\circ}$. Because no additional power is required, these passive films are desirable for applications in battery-powered displays. An optical model and a computer simulation have been developed by applying laws of optics in this paper. Simulation results show consistence with the experimental observations and it is concluded that for the brightness enhancement application, the optimal apex angle of micropyramids is $90^{\circ}$.

\section{Acknowledgments}

The authors would like to thank Drs W Bacher, M Heckele and $\mathrm{Mr}$ Biedermann at the Forschungszentrum research center, Karsruhe, Germany for valuable discussions and help in the laboratory hot embossing process. This project is supported in part by the Mechanical Industry Research Laboratories, Industrial Technology and Research Institute (ITRI), Hsin-Chu, Taiwan, a cooperation program between National Science Council, Taiwan and DFG, Germany, an NSF award (DMI-9800434) and a grant from the DARPA/ETO/MEMS program (F30602-98-2-01997).

\section{References}

[1] Peterson K E 1982 Silicon as a mechanical material Proc. IEEE 70 420-57

[2] Higuchi T and Yamagata Y 1993 Micro machining by machine tools Proc. IEEE Electro Mechanical Systems (MEMS93) pp 1-6

[3] Schubert K, Bier W, Linder G and Seidel D 1990 Profiled microdiamonds for producing microstructures Indust. Diamond Rev. 50 235-9

[4] 3M Company 1996 3M Brightness Enchancement Film 3M Center, St. Paul, MN

[5] Bacher W, Menz W and Mohr J 1995 The liga technique and its potential for microsystems-a survey IEEE Trans. Indust. Electron. 42 431-41

[6] Elders J, Jansen H V and Elwenspoek M 1995 Deemo: A new technology for the fabrication of microstructures Proc. IEEE Electro Mechanical Systems (MEMS95) pp 238-43

[7] Kohler U, Guber A E, Bier W and Heckele W 1996 Fabrication of microlenses by plasmaless isotropic etching combined with plastic moulding Sensors Actuators A 53 361-3

[8] Kohler U, Guber A E, Bier W, Heckele W and Schaller Th 1996 Fabrication of microlenses by combining silicon technology, mechanical micromachining and plastic molding Proc. SPIE 2687 18-22

[9] Larsson O, Ohman O, Billman A, Lundbladh L, Lindell C and Palmskog G 1997 Silicon based replication technology of 3d-microstructures by conventional cd-injection molding techniques Proc. 9th Int. Conf. on Solid-State Sensors and Actuators, Transducers'97 pp 1415-18

[10] Liwei Lin, Cheng Y T and Chiu C-J 1998 Comparative study of hot embossed microstructures fabricated by laboratory and commercial environments Microsyst. Technol. J. 4 113-16

[11] Olsson A et al 1997 Valve-less diffuser micropumps fabricated using thermoplastic replication Proc. IEEE Electro Mechanical Systems (MEMS97) pp 305-10

[12] Bassous B 1978 Fabrication of novel three-dimensional microstructures by the anisotropic etching of (100) and (110) silicon IEEE Trans. Electron Devices 25 1178-85

[13] Liwei Lin, Chiu C J, Bacher W and Heckle M 1996 Microfabrication using silicon mold inserts and hot embossing Proc. 6th Int. Symp. on Micro Machine and Human Science pp 67-71

[14] Hecht E 1989 Optics 2nd edn (Reading, MA: Addison-Wesley)

[15] Super Optics Development Co, Ltd 1986 Electro Luminescent Lamp (Tainan, Taiwan)

[16] Strandman C, Rosengren L, Elderstig H G A and Backlund Y 1995 Fabrication of $45^{\circ}$ mirrors together with well-defined v-grooves using wet anisotropic etching of silicon J. Microelectromech. Syst. 4 213-19 